\title{
Malaria mapping: understanding the global endemicity of falciparum and vivax malaria

\author{
Ursula Dalrymple ${ }^{\dagger}$, Bonnie Mappin $^{\dagger}$ and Peter W. Gething ${ }^{*}$
}

\begin{abstract}
The mapping of malaria risk has a history stretching back over 100 years. The last decade, however, has seen dramatic progress in the scope, rigour and sophistication of malaria mapping such that its global distribution is now probably better understood than any other infectious disease. In this minireview we consider the main factors that have facilitated the recent proliferation of malaria risk mapping efforts and describe the most prominent global-scale endemicity mapping endeavours of recent years. We describe the diversification of malaria mapping to span a wide range of related metrics of biological and public health importance and consider prospects for the future of the science including its key role in supporting elimination efforts.
\end{abstract}

Keywords: Malaria, Mapping, Modelling, Falciparum, Vivax, Model-based geostatistics

\section{Introduction}

Like most vector-borne diseases, malaria endemicity is partly determined by the local environment that houses its human and anopheline hosts and mediates the interactions between them. This environmental dependency leads to complex patterns of geographical variation in malaria transmission at almost every scale. Risk is rarelyuniform whether considered between households in a village, villages in a district or districts in a country [1]. The importance of evaluating local heterogeneity has motivated a long lineage of epidemiologists and disease control practitioners to generate maps of malaria risk to better understand local disease ecology and inform control activities [2]. The first serious attempt to audit the pattern of malaria endemicity at the global scale was undertaken in 1968 by Lysenko and Semashko [3]. This represented a major synthesis of historical records, maps of various malaria metrics (such as parasite rate, vector distributions, entomological inoculation rate, sickle cell incidence) and expert opinion and yielded a global map of malaria endemicity at the assumed peak of transmission intensity around the start of the 20th century. This map, stratified into four classes of endemicity, has since been digitised [4] and remains the most plausible

\footnotetext{
* Correspondence: peter.gething@zoo.ox.ac.uk

${ }^{\dagger}$ Equal contributors

Department of Zoology, Spatial Ecology and Epidemiology Group, University of Oxford, Tinbergen Building, Oxford, UK
}

reconstruction of global malaria risk in the largely preindustrial era and prior to widespread malaria control efforts [5] (Fig. 1).

It is now nearly half a century since the Lysenko map was published and, during most of that period, few efforts were made to improve on it. However, initiatives such as the continent-wide Mapping Malaria Risk in Africa/Atlas du Risque de la Malaria en Afrique (MARA/ARMA) project [6], instigated in 1997, and 8 years later the global Malaria Atlas Project (MAP) [7], catalysed a renaissance that has transformed the science of malaria risk mapping and its role in contemporary efforts to control, progressively eliminate and ultimately eradicate malaria.

In this minireview we present a condensed overview of: (i) the main factors that have facilitated the recent proliferation of malaria risk mapping efforts; (ii) prominent global-scale endemicity mapping endeavours of recent years; (iii) the diversification of malaria mapping to span a wide range of related metrics of biological and public health importance; and (iv) prospects for the future of the science including its key role in supporting elimination efforts.

\section{Enabling factors in the malaria mapping renaissance Increasing data availability}

Since the late 1980s, nationally representative crosssectional household surveys have been supported by a 


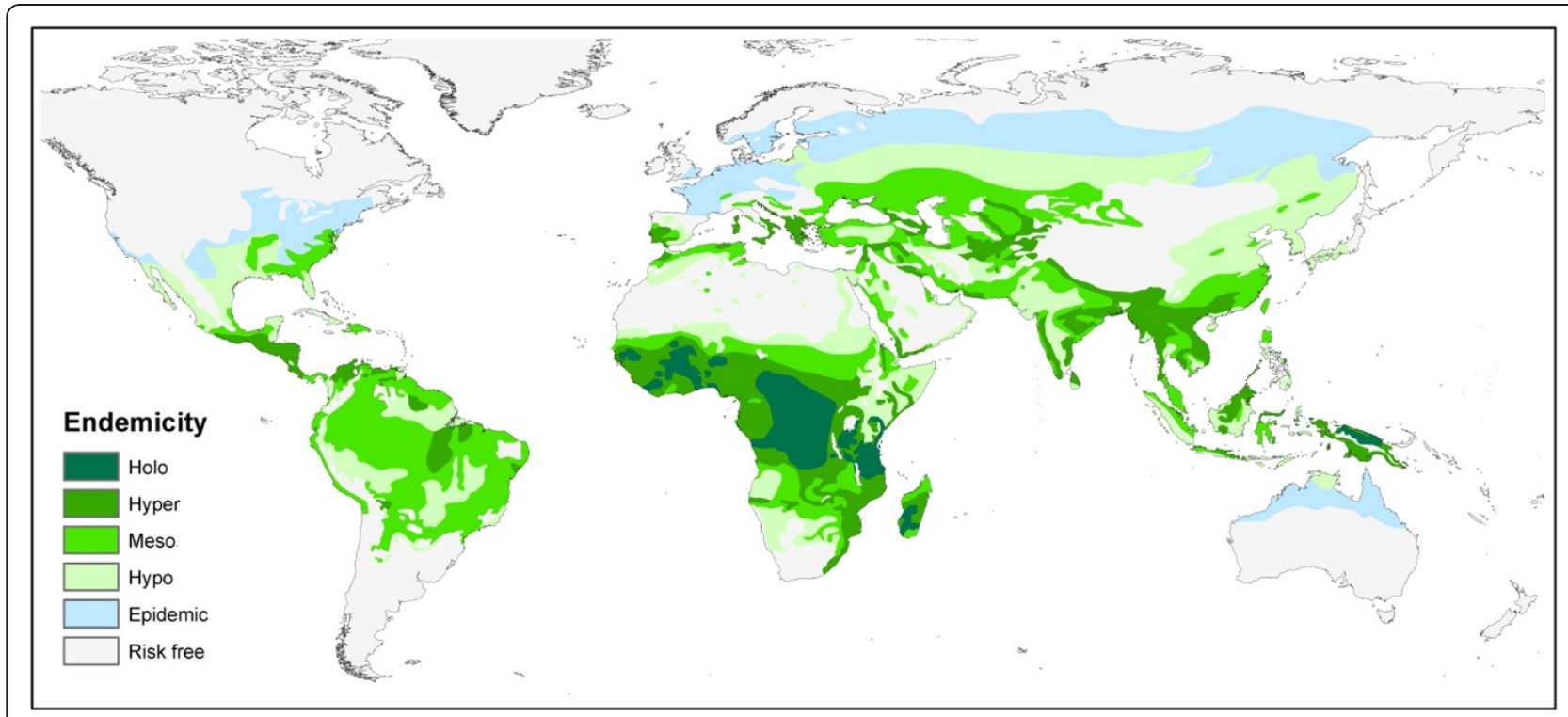

Fig. 1 The digitised 'Lysenko' map of global malaria endemicity circa 1900. This historic map constructed by Lysenko and Semashko [3] during the 1960s was based on a worldwide assembly of diverse malariometric data, simple climatic rules and expert opinion. The classic strata of malaria endemicity are described, each relating to infection prevalence (parasite rate, PR) in children: hypoendemic, PR $<10 \%$; mesoendemic, PR $\geq 10 \%$ and $<50 \%$; hyperendemic, PR $\geq 50 \%$ and $<75 \%$; and holoendemic, PR $\geq 75 \%$. This is a reproduction of the map in Hay et al. [4]

number of multilateral initiatives including the Demographic and Health Surveys (DHS) Program [8] and the UNICEF Multiple Indicator Cluster Survey (MICS) [9]. Such surveys have frequently been conducted in malaria-endemic countries and now include a growing suite of questions designed to gauge population access and use of malaria prevention, diagnostics and treatment. Since 2006, DHS surveys have begun to obtain blood samples from children under 5 years of age (and in some surveys, pregnant women) for parasite-based diagnosis of malaria using rapid diagnostic tests (RDTs) or microscopy. Crucially for mapping, these data tend to be accompanied by geographical coordinates denoting the location of the village or community from which each individual was sampled. These standardised and prospectively designed infection prevalence (or 'parasite rate') data are ideally suited as a basis for nationalscale endemicity mapping and have some key advantages over retrospective assemblies of ad hoc parasite rate data obtained, for example, from systematic literature searches. The influence of these large-scale programmes of national surveys, along with an increasing number of independent and nationally-led malaria indicator surveys, has transformed the availability of geolocated parasite rate data over the past decade (Fig. 2).

\section{Improved environmental covariates of malaria risk}

Along with data on malaria infection prevalence itself, a vital component of modern disease mapping methods is the inclusion of high quality data layers describing environmental or socio-demographic variables that correlate with malaria risk and can be used as empirical covariates. The ongoing refinement of satellite and airborne remote sensing platforms, and commensurate sophistication of post-processing algorithms and computational infrastructure for storage and dissemination of the resulting imagery, has led to a huge diversity of variables being included as part of spatial malaria models, as reviewed elsewhere [10]. In addition, work by malaria modelling groups has sought to modify existing geospatial variables to create malaria-specific products to enhance their utility for mapping. One recent example has been the manipulation of remotely-sensed data on landsurface temperature to create indices of temperature suitability for transmission of Plasmodium falciparum and Plasmodium vivax [11-13]. Recent work has also focused on the extension of traditionally static geospatial covariates into libraries of temporally dynamic data that potentially enable exploration of seasonal, inter-annual and long-term changes in environmental conditions on malaria transmission [10]. With the greater abundance of potential covariate layers has come an increased need for robust approaches to variable selection - allowing multivariate spatial models of malaria risk to be constructed that use an optimum set of covariates that maximise predictive power and avoid over-fitting the response data. Such approaches include Bayesian model selection procedures $[14,15]$ and exhaustive machine-learning techniques [10]. 


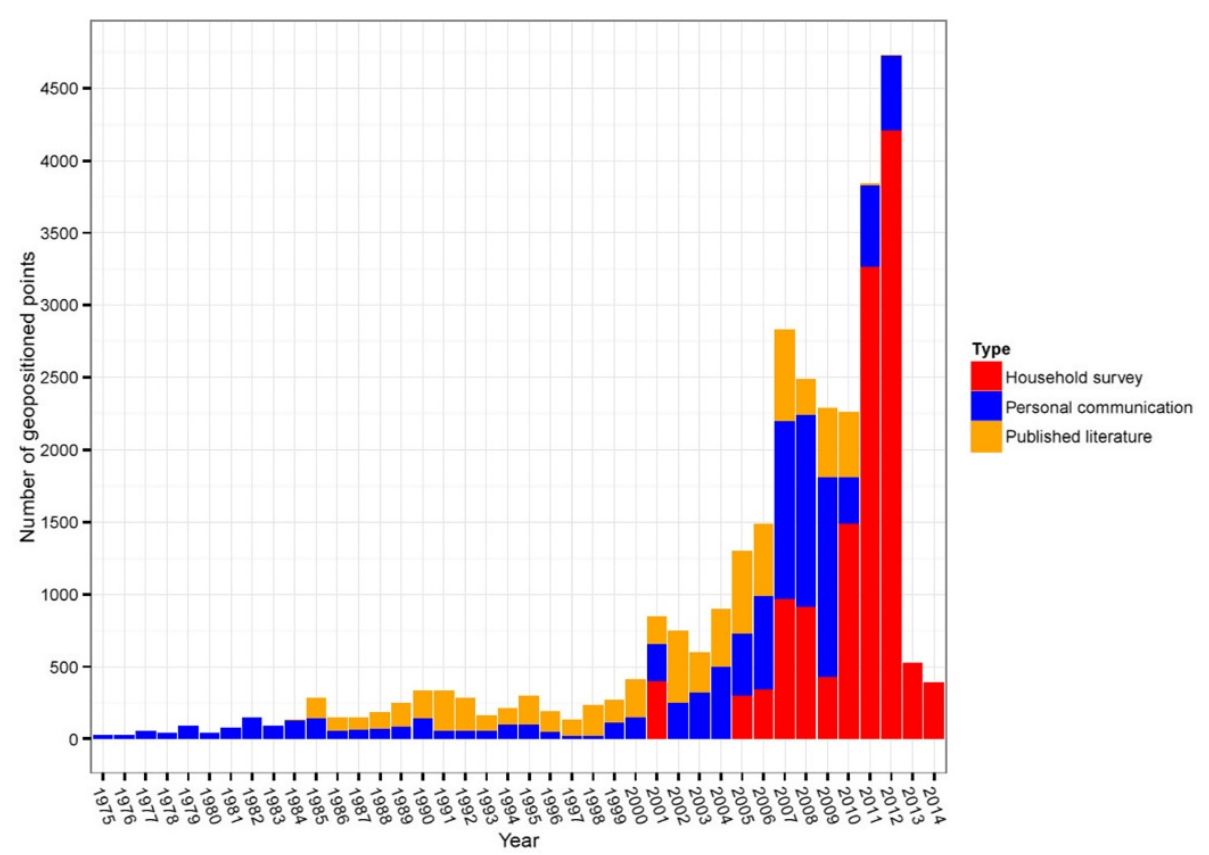

Fig. 2 Growth in the availability of georeferenced data on Plasmodium falciparum parasite rate. The data shown here represent the assembly for sub-Saharan Africa maintained by the Malaria Atlas Project, with an individual 'point' representing a spatially and temporally unique observation of community-level infection prevalence. The search methodology used to acquire the published literature and personal communication data points is described in detail elsewhere [67], and the household survey data points are collated from the sources described above and additional reports from national malaria control programmes. The reduced numbers in 2013 and 2014 are to be expected due to the lag time between data collection and its subsequent release

\section{Advances in analytical techniques}

The Lysenko map was constructed in the best traditions of manual cartography - with an emphasis on assimilating a wide variety of disparate data sources into a single synthesised map without any formal underlying quantitative framework. Whilst the result was an impressive summary of the state of knowledge existing at that time, this heuristic approach suffers a number of important drawbacks. Importantly, the likely accuracy of the map and how this varies from place to place can be neither measured nor communicated to end-users, placing a fundamental limitation on its use for critical public health decisions. In contrast, modern maps of malaria and other infectious diseases tend to result from formal spatial statistical models that aim to not only optimise accuracy but convey the spatially varying level of uncertainty associated with the mapped surface. The current state-of-the-art models tend to stem from a body of theory defined in the late 1990s known as model-based geostatistics (MBG) [16, 17]. MBG disease models, generally implemented in a Bayesian framework [16, 17], take point observations of disease prevalence from dispersed survey locations and generate interpolated estimates of prevalence at unsampled locations to generate continuous maps. Unlike simpler interpolation methods, MBG models capture both the inherent spatial structure displayed in a dataset (via a covariance function) and the uncertainty around that structure. They also provide a natural framework for incorporation of multivariate relationships with covariates, and the use of disease response data in continuous, count or proportion format with appropriate models for sampling error. Since such techniques were first demonstrated in a malaria mapping context [18], many useful elaborations have been developed. Gosoniu et al. $[15,19]$ demonstrated an approach to allow non-stationarity enabling the spatial structure of the model to vary from place to place to better capture local variation when modelling over large areas. MBG techniques have been extended to map malaria both spatially and temporally [20], allowing data from multiple time points to contribute appropriately to a single cross-sectional map [21-23] and, more recently, to explore spatio-temporal patterns of change through time [24]. Gething et al. [25] introduced the ability to quantify aggregated uncertainty over space and time in a global-scale MBG model with use of an approximating joint simulation algorithm. This allowed predicted malaria risk levels to be summarised formally at the varying scales of geographical aggregation over which public health decisions are usually made.

Contemporary maps of continental and global endemicity Numerous studies have developed Bayesian geostatistical models to create national or multi-national maps of 
malaria risk, often intended to aid national malaria control programme policy decisions in specific regions and improve understanding of within-country patterns of spatial heterogeneity in malaria transmission and burden [26-38]. Additionally, spatial scanning methods to detect clusters (or 'hotspots') of intense malaria transmission at very fine spatial scales have been developed and applied at a sub-national scale. These methods can be used to identify individual homesteads within hotspots with particularly intense malaria transmission $[1,39]$.

The first attempt to map malaria endemicity at global scales using MBG techniques was completed for P. falciparum in 2009 by the Malaria Atlas Project [23]. This was the culmination of 5 years of data assembly [40], delineation of the limits of stable transmission [41], and methodological development to extend existing MBG approaches to incorporate additional functionality including an embedded age-prevalence standardization model [42] and the incorporation of the spherical shape of the Earth within the model computation. The resulting map, describing infection prevalence in 2-10 year olds across a $5 \times 5 \mathrm{~km}$ resolution grid, was the first global assessment of malaria risk that used a standardised data and modelling framework and was able to provide accompanying maps describing the geographically varying uncertainty associated with each predicted pixel value. While the 2007 map marked a new era in global malaria cartography, the field continued to evolve rapidly and an updated map was generated for the year 2010 (Fig. 3a) in which, along with a large influx of new PfPR surveys, some important methodological advances were made [21]. Early computational constraints in the implementation of MBG at the global scale meant that the earlier map included no environmental covariates with the exception of urbanity. For the 2010 iteration, a more efficient MCMC algorithm allowed the multivariate effects on PfPR of a wider suite of 20 environmental and socio-demographic covariates to be incorporated, substantially improving predictive accuracy and the level of spatial detail that could be resolved.

In that same year, a corresponding global endemicity map of $P$. vivax prevalence was produced by the Malaria Atlas Project [22] (Fig. 3b). While the basic geostatistical architecture mirrored that developed for P. falciparum, the unique epidemiology [43] of this less well studied parasite species posed unique challenges for global mapping. A particular challenge was the handling of data in Africa, where a commonly held mantra is that $P$. vivax is absent due to the near-fixation in the population of Duffy negativity - a genetic blood disorder that conveys near total protection from $P$. vivax infection. A detailed literature review, however, demonstrated the presence of the parasite in nearly all Africa countries. Rather than branding large swathes of the continent as being vivaxfree, a more elaborate approach was developed that drew from data on both $P v \mathrm{PR}$ and population prevalence of the Duffy genetic trait [44] such that both quantities could be mapped simultaneously. These works paved the way to a comprehensive review of the global public health significance of $P$. vivax, which suggested that morbidity and fatalities caused by $P$. vivax may be substantially underestimated [45].

These maps provided new benchmark evaluations of the contemporary landscape of malaria risk worldwide. For the first time, international policy makers could draw upon a standardised methodology that allowed meaningful country-to-country comparison of malaria risk exposure for national populations. Since the publication of these 2010 maps, there has been an increasing interest in extending the methodologies to allow prediction of risk both geographically and through time, motivated in part by the need to evaluate progress towards international targets set for the year 2015 [46]. By mapping malaria risk dynamically, patterns of change can be explored. In 2014, Noor et al. produced an analysis of changing risk of $P$. falciparum malaria across Africa since 2000 [47]. This analysis used a large assembly of PfPR data collected over the preceding decades, along with four static environmental covariates (precipitation, temperature suitability index, enhanced vegetation index and urbanisation) in an MBG model to generate cross-sectional risk maps for the years 2000, 2005 and 2010 [47]. This analysis allowed the first formal insights into patterns of changing malaria in Africa since the turn of the millennium, a period of major international efforts to raise funding and scale up control efforts. The study described evidence of declining PfPR in nearly all endemic Africa countries between 2000 and 2010, but in many cases these declines were relatively modest.

A more recent study by the Malaria Atlas Project has also sought to evaluate changing risk patterns in Africa, for the more recent year of 2015, with some important differences in input data and approach (Bhatt S, Weiss DJ, Mappin B, Dalrymple U, Cameron E, Bisanzio D, et al: Insecticide-treated nets (ITNs) in Africa 2000-2017: coverage, system efficiency and future needs to achieve international targets, unpublished). Most significant was the development and incorporation of detailed spatiotemporal reconstructions of coverage patterns for the major malaria control interventions over the same time period [24]: insecticide-treated bed nets (ITNs); indoor residual spraying (IRS); and artemisinin-based combination therapy (ACT) antimalarials for malaria case management. Incorporating these within a space-time MBG framework allowed improved estimates of infection prevalence through time. By also including a wide range of temporally dynamic background covariates since 2000 [10], it was possible to disentangle the relative contributions of each intervention to the observed declines in PfPR, in the context of any 


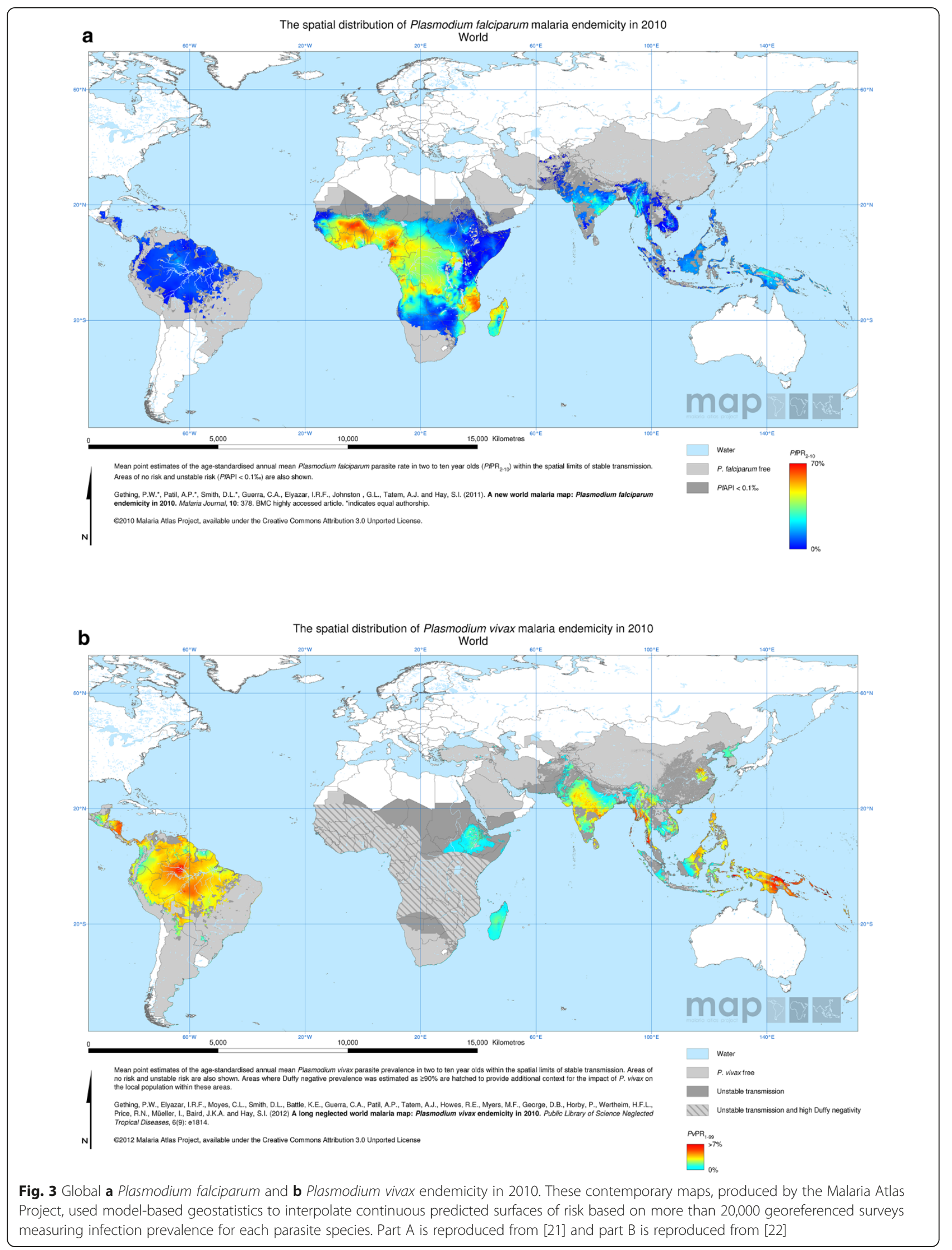


changes in underlying environmental conditions. This work provided important evidence on the impact of large-scale control efforts in Africa since the turn of the Millennium.

\section{Beyond prevalence: the diversification of malaria mapping \\ Deriving malariometrics from parasite rate estimates}

The developments in mapping malaria infection prevalence, described above, have spawned a larger and more diverse body of work, allowing maps of parasite rate to be used as an input to predict the distribution of a wide range of other malariometrics with distinct utility to biologists, epidemiologists and decision-makers. These have included the mapping of clinical incidence rates, entomological inoculation rates (EIRs), the basic reproductive number $(R)$ and the burden of morbidity due to malaria in pregnancy.

The measurement of malaria incidence (the number of clinical cases that occur annually within a given population) is typically measured by one of two approaches: either by using direct data on observed cases detected via routine surveillance systems; or by using maps of infection prevalence and using a model to convert this metric into a plausible value of clinical incidence at each mapped location (known as the 'cartographic' approach). In much of sub-Saharan Africa, and in particular those countries with the higher malaria burdens, routine surveillance data are not considered sufficiently robust to use as a basis for estimating clinical incidence or evaluating trends through time [24]. The development of continuous parasite rate maps has made it possible to model statistically the relationship between $P$. falciparum prevalence and clinical incidence rates. Initial efforts to construct a PfPR-incidence relationship for $P$. falciparum burden estimation used data-driven fits with varying sophistication from first-order stratification by endemicity class to hierarchical Gaussian process regression [48-50], and projections based on the calibration of a steady-state compartmental transmission model [51]. In 2015, Cameron et al. used three of the most contemporary published prevalence-incidence models were calibrated against a purpose-built dataset of incidence counts from numerous sites across sub-Saharan Africa (Cameron E, Battle KE, Bhatt S, Weiss DJ, Bisanzio D, Dalrymple U, et al.: Defining the relationship between infection prevalence and clinical incidence of Plasmodium falciparum malaria: an ensemble model, Submitted). The combined predictive power of this ensemble model allowed forecasts of expected malaria incidence with limited uncertainty, and highlighted general conceptual agreement between the models. The ensemble model has since been utilised, alongside the Malaria Atlas Project's estimations of yearly $P f P R$, to estimate the changing incidence of $P$. falciparum malaria from 2000 to 2015.

The EIR, or entomological inoculation rate, describes the number of expected bites from infected mosquitoes per person per unit time and is often used as a standardised measure of transmission intensity [21]. Work has been done to assemble observations of EIR across Africa and define their relationship with PfPR [52]. In an analogous way to the cartographic estimation of clinical incidence, this has allowed maps of infection prevalence to be converted into maps of EIR, describing this key entomological quantity geographically across the endemic world [21]. The same work also included a model to extend PfPR maps to describe the global distribution of the basic reproductive number, $R$, for $P$. falciparum malaria. $R$ quantifies the potential of $P$. falciparum to spread throughout a population (formally the number of new cases arising per index case per generation of the parasite) and provides important insights into, for example, the magnitude of impact that control efforts must have at each location in order to drive transmission towards elimination.

Estimates of the number of pregnant women at risk of malaria infection globally have been made [53] by combining national estimates of numbers of pregnancies for 2007 and MAP's 2007 and 2003 estimates of global $P$. falciparum [41] and $P$. vivax endemicity [54], respectively. Although the World Health Organization (WHO) estimates annually the number of pregnant women at risk of malaria in Africa, this study provided the first comprehensive and contemporary estimation of the number of pregnancies at risk of malaria outside of Africa.

\section{Mapping for elimination}

Long-term international policy around malaria control is increasingly reoriented to achieve progressive elimination of malaria country-by-country with the ultimate goal of reaching eradication of the disease [55,56]. An initial utility of global endemicity maps in this context has been as one component of a wider assessment of relative elimination feasibility between countries, helping guide prioritisation and target-setting [57]. As more malaria-endemic countries enter the elimination phase, new challenges arise for malaria cartography to provide geospatial information tailored to the distinct operational requirements of elimination activities. An immediate technical challenge arises from the difficulty in obtaining useful metrics of malaria transmission at very low levels of transmission. Traditional parasite rate surveys become underpowered to detect very rare infections, and research is underway to examine a range of alternative metrics for mapping, including molecular-based parasite detection or identification of serological markers of infection exposure [58-60]. In elimination scenarios, the diagnostic accuracy of response data becomes more important in order to detect subpatent infections which are thought to account for $20-50 \%$ of human-to-mosquito transmissions in low endemicity areas [61]. Investment in more sensitive 
case detection methods is required to accurately assess transmission intensity [62]. Additionally, methods to standardise diagnostic data inputs prior to mapping are required to eliminate uncertainty, especially in elimination areas. Regression models have been developed in recent years between both microscopy and PCR [61], and RDT and microscopy [63]. These models can be applied to observed prevalence measured by one diagnostic test in an elimination area to estimate the expected observed prevalence using an alternative method of diagnosis. Additionally, measuring progress towards elimination is aided by the ever-increasing availability of map data and measurements of parasite rate over time and space which can be used for comparison.

Further challenges in defining geographic patterns of risk arise from the issue of human movement. When cases become rare, the relative contribution of imported malaria - infections originating outside the eliminating country - tends to increase until they can become the primary reason for transmission being sustained. This complicates the description of risk patterns and necessitates an understanding of human movement alongside data on observed infections. In a study in Namibia, Tatem et al. integrated mobile phone data (which can serve as a proxy for human movement patterns) with case-based risk maps to predict hotspots of transmission in generally lowtransmission settings [64]. Other work has sought to use a range of input metrics and mapping techniques to identify the fine-scale or seasonal variations in risk which become important in understanding the highly heterogeneous pattern of risk in elimination settings $[65,66]$.

\section{Conclusions}

This review has summarised the evolution of malaria risk mapping over the past decade and the improvements in data availability, computational power and methodological developments that have facilitated it. This ongoing development has transformed malaria risk mapping from an art to a science, and can now bring mature and statistically robust approaches to bear on a diverse range of cartographic questions. As the global malaria landscape continues to change over the coming years, these geospatial approaches must continue to evolve in order to provide accurate descriptions of change, insight into the many factors driving those changes and, ultimately, to continue to contribute to evidence-based malaria control and elimination activities worldwide.

\section{Abbreviations}

ACT: Artemisinin-based combination therapy; DHS: Demographic and Health Surveys; EIR: Entomological inoculation rate; IRS: Indoor residual spraying; ITN: Insecticide-treated bed net; MAP: Malaria Atlas Project; MARA/ARMA: Mapping Malaria Risk in Africa/Atlas du Risque de la Malaria en Afrique; MBG: Model-based geostatistics; MICS: Multiple Indicator Cluster Survey; PCR: Polymerase chain reaction; PR: Parasite rate; RDT: Rapid diagnostic test; WHO: World Health Organization
Competing interests

The authors declare that they have no competing interests.

\section{Authors' contributions}

UD and BM reviewed the existing literature and drafted the manuscript. $P G$ reviewed the manuscript. All authors read and approved the final manuscript.

Received: 17 March 2015 Accepted: 18 May 2015

Published online: 12 June 2015

\section{References}

1. Bejon P, Williams TN, Nyundo C, Hay SI, Benz D, Gething PW, et al. A micro-epidemiological analysis of febrile malaria in Coastal Kenya showing hotspots within hotspots. Elife. 2014;3, e02130.

2. Mouchet J, Carnevale P, Coosemans M, Julvez J, Manguin S, Richard-Lenoble D, et al. Biodiversité du paludisme dans le monde. Paris: John Libbey Eurotext; 2004.

3. Lysenko AJ, Semashko IN. Geography of malaria. A medico-geographic profile of an ancient disease. Itogi Nauk Med Geogr. 1968;25:146.

4. Hay SI, Guerra CA, Tatem AJ, Noor AM, Snow RW. The global distribution and population at risk of malaria: past, present, and future. Lancet Infect Dis. 2011;4:327-36

5. Gething PW, Smith DL, Patil AP, Tatem AJ, Snow RW, Hay SI. Climate change and the global malaria recession. Nature. 2010;465:342-5.

6. Le Sueur D, Binka F, Lengeler C, de Savigny D, Snow B, Teuscher T, et al. An atlas of malaria in Africa. Afr Health. 1997;19:23-4.

7. Hay SI, Snow RW. The Malaria Atlas Project: developing global maps of malaria risk. PLoS Med. 2006;3, e473.

8. DHS Program. [http://dhsprogram.com/]

9. UNICEF MICS. [http://www.childinfo.org/mics_available.html]

10. Weiss DJ, Mappin B, Dalrymple U, Bhatt S, Cameron E, Hay SI, et al. Re-examining environmental correlates of Plasmodium falciparum malaria endemicity: a data-intensive variable selection approach. Malar J. 2015;14:68.

11. Weiss DJ, Bhatt S, Mappin B, Van Boeckel TP, Smith DL, Hay SI, et al. Air temperature suitability for Plasmodium falciparum malaria transmission in Africa 2000-2012: a high-resolution spatiotemporal prediction. Malar J. 2014;13:171

12. Garske T, Ferguson NM, Ghani AC. Estimating air temperature and its influence on malaria transmission across Africa. PLoS One. 2013;8, e56487.

13. Gething PW, Van Boeckel TP, Smith DL, Guerra CA, Patil AP, Snow RW, et al. Modelling the global constraints of temperature on transmission of Plasmodium falciparum and P. vivax. Parasit Vectors. 2011;4:92.

14. Vounatsou P, Raso G, Tanner M, N'goran EK, Utzinger J. Bayesian geostatistical modelling for mapping schistosomiasis transmission. Parasitology. 2009;136:1695-705.

15. Gosoniu L, Vounatsou P, Sogoba N, Smith T. Bayesian modelling of geostatistical malaria risk data. Geospat Health. 2006;1:127-39.

16. Diggle PJ, Tawn JA, Moyeed RA. Model-based geostatistics. J R Stat Soc Ser C Appl StaT. 1998;47:299-350.

17. Diggle P, Ribeiro P. Model-based geostatistics. New York: Springer; 2007. p. 246.

18. Diggle P, Moyeed R, Rowlingson B, Thomson M. Childhood malaria in the Gambia: a case-study in model-based geostatistics. J R Stat Soc. 2002;51:493-506.

19. Gosoniu L, Vounatsou P, Sogoba N, Maire N, Smith T. Mapping malaria risk in West Africa using a Bayesian nonparametric non-stationary model. Comput Stat Data Anal. 2009:53:3358-71.

20. Gething PW, Noor AM, Gikandi PW, Ogara EAA, Hay SI, Nixon MS, et al. Improving imperfect data from health management information systems in Africa using space-time geostatistics. PLoS Med. 2006;3, e271.

21. Gething PW, Patil AP, Smith DL, Guerra CA, Elyazar IRF, Johnston GL, et al. A new world malaria map: Plasmodium falciparum endemicity in 2010. Malar J. 2011;10:378.

22. Gething PW, Elyazar IRF, Moyes CL, Smith DL, Battle KE, Guerra CA, et al. A long neglected world malaria map: Plasmodium vivax endemicity in 2010. PLoS Negl Trop Dis. 2012;6, e1814.

23. Hay SI, Guerra CA, Gething PW, Patil AP, Tatem AJ, Noor AM, et al. A world malaria map: Plasmodium falciparum endemicity in 2007. PLoS Med. 2009;6, e1000048.

24. World Health Organization. World Malaria Report 2014. Geneva: World Health Organization; 2014. 
25. Gething PW, Patil AP, Hay SI. Quantifying aggregated uncertainty in Plasmodium falciparum malaria prevalence and populations at risk via efficient space-time geostatistical joint simulation. PLoS Comput Biol. 2010;6, e1000724.

26. Kazembe LN, Kleinschmidt I, Holtz TH, Sharp BL. Spatial analysis and mapping of malaria risk in Malawi using point-referenced prevalence of infection data. Int J Health Geogr. 2006;5:41.

27. Noor AM, Clements ACA, Gething PW, Moloney G, Borle M, Shewchuk T, et al. Spatial prediction of Plasmodium falciparum prevalence in Somalia. Malar J. 2008;7:159.

28. Elyazar IRF, Gething PW, Patil AP, Rogayah H, Kusriastuti R, Wismarini DM, et al. Plasmodium falciparum malaria endemicity in Indonesia in 2010. PLoS One. 2011;6, e21315.

29. Elyazar IRF, Gething PW, Patil AP, Rogayah H, Sariwati E, Palupi NW, et al. Plasmodium vivax malaria endemicity in Indonesia in 2010. PLoS One. 2012; 7, e37325.

30. Raso G, Schur N, Utzinger J, Koudou BG, Tchicaya ES, Rohner F, et al. Mapping malaria risk among children in Côte d'Ivoire using Bayesian geo-statistical models. Malar J. 2012;11:160

31. Gosoniu L, Msengwa A, Lengeler C, Vounatsou P. Spatially explicit burden estimates of malaria in Tanzania: Bayesian geostatistical modeling of the malaria indicator survey data. PLoS One. 2012;7, e23966.

32. Riedel N, Vounatsou P, Miller JM, Gosoniu L, Chizema-Kawesha E, Mukonka $\checkmark$, et al. Geographical patterns and predictors of malaria risk in Zambia: Bayesian geostatistical modelling of the 2006 Zambia national malaria indicator survey (ZMIS). Malar J. 2010;9:37.

33. Gosoniu L, Veta AM, Vounatsou P. Bayesian geostatistical modeling of Malaria Indicator Survey data in Angola. PLoS One. 2010;5, e9322.

34. Noor AM, Uusiku P, Kamwi RN, Katokele S, Ntomwa B, Alegana VA, et al. The receptive versus current risks of Plasmodium falciparum transmission in Northern Namibia: implications for elimination. BMC Infect Dis. 2013;13:184

35. Reid H, Haque U, Clements ACA, Tatem AJ, Vallely A, Ahmed SM, et al. Mapping malaria risk in Bangladesh using Bayesian geostatistical models. Am J Trop Med Hyg. 2010;83:861-7.

36. Noor AM, ElMardi KA, Abdelgader TM, Patil AP, Amine A, Bakhiet S, et al. Malaria risk mapping for control in the republic of Sudan. Am J Trop Med Hyg. 2012;87:1012-21.

37. Bennett A, Kazembe L, Mathanga DP, Kinyoki D, Ali D, Snow RW, et al. Mapping malaria transmission intensity in Malawi, 2000-2010. Am J Trop Med Hyg. 2013:89:840-9.

38. Noor AM, Gething PW, Alegana VA, Patil AP, Hay SI, Muchiri E, et al. The risks of malaria infection in Kenya in 2009. BMC Infect Dis. 2009;9:180.

39. Bejon P, Williams TN, Liljander A, Noor AM, Wambua J, Ogada E, et al. Stable and unstable malaria hotspots in longitudinal cohort studies in Kenya. PLoS Med. 2010;7, e1000304

40. Guerra CA, Hay SI, Lucioparedes LS, Gikandi PW, Tatem AJ, Noor AM, et al. Assembling a global database of malaria parasite prevalence for the Malaria Atlas Project. Malar J. 2007:6:17.

41. Guerra CA, Gikandi PW, Tatem AJ, Noor AM, Smith DL, Hay SI, et al. The limits and intensity of Plasmodium falciparum transmission: implications for malaria control and elimination worldwide. PLoS Med. 2008;5, e38.

42. Smith DL, Guerra CA, Snow RW, Hay SI. Standardizing estimates of the Plasmodium falciparum parasite rate. Malar J. 2007;6:131.

43. Mueller I, Galinski MR, Baird JK, Carlton JM, Kochar DK, Alonso PL, et al. Key gaps in the knowledge of Plasmodium vivax, a neglected human malaria parasite. Lancet Infect Dis. 2009;9:555-66.

44. Howes RE, Patil AP, Piel FB, Nyangiri OA, Kabaria CW, Gething PW, et al. The global distribution of the Duffy blood group. Nat Commun. 2011;2:266.

45. Battle KE, Gething PW, Elyazar IRF, Moyes CL, Sinka ME, Howes RE, et al. The global public health significance of Plasmodium vivax. Adv Parasitol. 2012:80:1-111.

46. Partnership RBM. Global Malaria Action Plan 1 (2000-2015). Geneva: World Health Organization; 2008.

47. Noor AM, Kinyoki DK, Mundia CW, Kabaria CW, Mutua JW, Alegana VA, et al. The changing risk of Plasmodium falciparum malaria infection in Africa: 2000-10: a spatial and temporal analysis of transmission intensity. Lancet. 2014;383:1739-47.

48. Cibulskis RE, Aregawi M, Williams R, Otten M, Dye C. Worldwide incidence of malaria in 2009: estimates, time trends, and a critique of methods. PLoS Med. 2011;8, e1001142.

49. Patil AP, Okiro EA, Gething PW, Guerra CA, Sharma SK, Snow RW, et al. Defining the relationship between Plasmodium falciparum parasite rate and clinical disease: statistical models for disease burden estimation. Malar J. 2009;8:186.

50. Hay SI, Okiro EA, Gething PW, Patil AP, Tatem AJ, Guerra CA, et al. Estimating the global clinical burden of Plasmodium falciparum malaria in 2007. PLoS Med. 2010;7, e1000290.

51. Griffin JT, Ferguson NM, Ghani AC. Estimates of the changing age-burden of Plasmodium falciparum malaria disease in sub-Saharan Africa. Nat Commun. 2014;5:3136.

52. Smith DL, Dushoff J, Snow RW, Hay SI. The entomological inoculation rate and Plasmodium falciparum infection in African children. Nature. 2005:438:492-5.

53. Dellicour S, Tatem AJ, Guerra CA, Snow RW, Ter Kuile FO. Quantifying the number of pregnancies at risk of malaria in 2007: a demographic study. PLoS Med. 2010;7, e1000221.

54. Guerra CA, Snow RW, Hay SI. Defining the global spatial limits of malaria transmission in 2005. Adv Parasitol. 2006;62:157-79.

55. Partnership RBM. Global Malaria Action Plan 2 (2016-2030). Geneva: World Health Organization; 2015.

56. World Health Organization. Draft Global Technical Strategy for Malaria 2016-2030. Geneva: World Health Organization; 2014.

57. Feachem RGA, Phillips AA, Hwang J, Cotter C, Wielgosz B, Greenwood BM, et al. Shrinking the malaria map: progress and prospects. Lancet. 2010;376:1566-78.

58. Hsiang MS, Hwang J, Kunene S, Drakeley C, Kandula D, Novotny J, et al. Surveillance for malaria elimination in Swaziland: a national cross-sectional study using pooled PCR and serology. PLoS One. 2012;7, e29550.

59. Tusting LS, Bousema T, Smith DL, Drakeley C. Measuring changes in Plasmodium falciparum transmission: precision, accuracy and costs of metrics. Adv Parasitol. 2014;84:151-208.

60. Okebe J, Affara M, Correa S, Muhammad AK, Nwakanma D, Drakeley C, et al. School-based countrywide seroprevalence survey reveals spatial heterogeneity in malaria transmission in the Gambia. PLoS One. 2014;9, e110926.

61. Okell LC, Bousema T, Griffin JT, Ouédraogo AL, Ghani AC, Drakeley CJ. Factors determining the occurrence of submicroscopic malaria infections and their relevance for control. Nat Commun. 2012;3:1237.

62. Moonen B, Cohen JM, Snow RW, Slutsker L, Drakeley C, Smith DL, et al. Operational strategies to achieve and maintain malaria elimination. Lancet. 2010;376:1592-603.

63. Mappin B, Dalrymple U, Cameron E, Bhatt S, Weiss DJ, Gething PW. Comparing community $P$. falciparum infection prevalence measured via microscopy versus rapid diagnostic test. Malar J. 2014;13:P60.

64. Tatem AJ, Huang Z, Narib C, Kumar U, Kandula D, Pindolia DK, et al. Integrating rapid risk mapping and mobile phone call record data for strategic malaria elimination planning. Malar J. 2014;13:52.

65. Cohen JM, Dlamini S, Novotny JM, Kandula D, Kunene S, Tatem AJ. Rapid case-based mapping of seasonal malaria transmission risk for strategic elimination planning in Swaziland. Malar J. 2013;12:61.

66. Sturrock HJW, Cohen JM, Keil P, Tatem AJ, Le Menach A, Ntshalintshali NE, et al. Fine-scale malaria risk mapping from routine aggregated case data. Malar J. 2014;13:421.

67. Gething PW, Patil AP, Smith DL, Guerra CA, Elyazar IRF, Johnston GL, et al. A new world malaria map: Plasmodium falciparum endemicity in 2010. Additional file A2 - Updates to the Plasmodium falciparum parasite rate survey database. Malar J. 2010;10:378. 